\title{
Infants' Perception of Dynamic Relations Between Objects: Passing Through or Support?
}

\author{
Margriet M. Sitskoorn and Ad W. Smitsman \\ University of Nijmegen
}

\begin{abstract}
This study investigated 4-, 6-, and 9-month-old infants' ability to perceive whether the width relation between a block and the opening of a box specified passing through or support. If infants could perceive which outcome was specified by the width relation, longer looking times would be expected for an outcome that violates this specification. Six- and 9-month-olds looked significantly longer when a block wider than a box opening passed through this opening and when a block with a width smaller than the opening seemed to become supported at the level of this opening. Results indicate that around 6 months of age, infants become able to perceive relations between objects that specify the outcomes of events.
\end{abstract}

Events consisting of interacting objects are a daily part of infants' lives. Colliding, cutting, containing, and supporting are examples of such interactions. When objects interact, relations between properties of objects constrain the course and the outcome of such interactions. These relations, which we call $d y$ namic object relations, are invariant properties of the event. That is, the course and outcome of an interaction covary with the dynamic relations between the objects involved. Perception of these relations allows for anticipation of the outcome of the event. The present study investigated infants' ability to perceive dynamic relations between objects.

According to Gibsonian theory (cf. J. J. Gibson, 1979), relations between objects can be perceived to the extent that information that specifies these relations is available for the perceptual system. For the visual system, information is contained within invariances in the flow of stimulation in the optic array (cf. J. J. Gibson, 1979). For example, a series of projective transformations in the visual field produces invariances that specify an object's shape, whereas its shape would remain ambiguous from any single projection (Ruff, 1982). Research on infant perception has shown that infants are able to perceive

Margriet M. Sitskoorn and Ad W. Smitsman, University of Nijmegen, Nijmegen, The Netherlands.

We thank The Netherlands' Organization for Scientific Research for a travel grant. Portions of the research reported in this article were presented in August 1990 at the Fourth European Conference on Developmental Psychology in Stirling, Scotland, and in July 1991 at the biennial meetings in Minneapolis, Minnesota.

We are indebted to Michael Czarnota, Frits Dexel, Michael Katzko, Cornelis van Lieshout, Erik van Loosbroek, Patrick Maitimo, Chiara Pisanu, Marianne Riksen-Walraven, and Roelof Schellingerhout for providing assistance during different phases of the research. We also thank the participating parents and their infants.

Correspondence concerning this article should be addressed to Margriet M. Sitskoorn, University of Nijmegen, Psychologisch Laboratorium, Postbus 9104,6500 HE Nijmegen, The Netherlands. Electronic mail may be sent via Internet to U212232@VM.UCI.KUN.NL. invariances that specify properties of a single object. There is evidence that on the basis of invariances, infants can perceive shape (Day \& McKenzie, 1973; E. J. Gibson, Owsley, Walker, \& Megaw-Nyce, 1979; Kellman, 1984; Ruff, 1982), size (Day \& McKenzie, 1981), and motion (E. J. Gibson, Owsley, \& Johnston, 1978; Ruff, 1985).

Invariances are not only induced by the properties of a single moving object but also by the dynamic relations between the properties of interacting objects. A dynamic relation will cause a specific transformation in the structure of the optic array. Perception of this transformation makes it possible to recognize that the relation is an invariant property of the interaction, irrespective of the objects involved in that interaction. If perception of this invariant property precedes the outcome of the interaction, it allows for anticipation of this outcome. Perceptual development involves an improvement in the ability to detect these kinds of invariances and therefore an increasing ability to anticipate event outcomes.

In the present study, we used 4-, 6-, and 9-month-old infants in four experiments to investigate this developing ability. We were specifically interested in the age at which infants were able to perceive whether the relation between the width of a block and the width of the opening of a box allowed the block to pass through the opening of the box or to become supported by its edges.

We chose passing through and support for several reasons. Both outcomes occur frequently in the daily life of infants. Many object functions and event outcomes are rooted in passing through and support, for example, using a box as a container and using the container as a carrier. At about 9 months of age, infants begin to engage in the production of these kinds of events themselves (Uzgiris, 1972), and by the end of the first year, infants explore the affordances of concavities even more thoroughly (Piaget, 1937/1954; Pieraut-Le Bonniec, 1985; Stambak, Sinclair, Verba, Moreno, \& Rayna, 1989).

If the width of an object is less than the width of the opening of a box, the block can pass through the opening and become contained by the box. If the width of the object is larger than the 
width of the opening of the box, the object cannot pass through the opening of the box but can become supported by the rims of the box. Because both passing through and support are determined by width relation, we were able to (a) investigate infants' perception of width relation with respect to two different event outcomes and (b) design our experiments in such a way that the outcomes of two different experiments were complementary to each other.

Previous studies have shown that infants already have some knowledge of passing through. Pieraut-Le Bonniec (1984, 1985 ) presented 7 - to 11 -month-old infants with tumblers that were open at the top, closed at the top by an opaque disk, or closed at the top by a transparent plastic plate. Ten-month-old infants inserted their hand into the open tumbler, whereas younger infants just grasped the rims. Nine-month-olds' exploration suggested that they were trying to understand the discrepancy between the visual and tactile properties of the tumblers. These data suggest that from 9 months of age, infants recognize that an opening allows passing through. Caron, Caron, and Antell (1988) and Maclean and Schuler (1989) investigated a combination of passing through and support. Caron et al. showed a group of 11-, 14-, 17-, and 20-month-old infants alternating video episodes of a container that contained (nonviolation) or failed to contain (violation) sand that was poured into it. Another group of infants saw alternations of a tube that failed to contain (nonviolation) or contained (violation) sand that was poured into it. Results showed that before 17 months of age, infants perceive that openings in surfaces of objects allow insertion, but it is not until after 17 months of age that infants realize that passageways must have supporting bottoms in order to contain ( see also Maclean \& Schuler, 1989).

Even though we used containers in our experiments, we did not investigate whether infants perceived the supportive function of the bottoms of the container but rather whether they perceived whether the rims of the box would provide support to an object. Some knowledge about infants' perception of these kind of support events can be gained from other studies on support. Keil (1979) found that 18-to 30-month-olds expected an object to fall only if it was completely unsupported. In Keil's experiment, one block was centered between and supported by two other blocks. When these two supporting blocks were both removed, infants showed surprise when the top block remained in place. In a second experiment, a fourth block was placed upright at the center of the top block and only the right support block was removed. Infants showed no surprise when the two blocks, though inadequately supported, remained in place.

The results of a study by Baillargeon and Hanko-Summers (1990) indicated that 7.5- to 9.5-month-old infants were able to distinguish between adequate and inadequate support. However, that ability was restricted to events that involved symmetrical objects. Infants did not perceive that an asymmetrical object will fall if its center of mass is not supported.

None of the studies described earlier provides evidence for infants' anticipation of passing through and support on the basis of perception of width relation. However, some evidence is provided by affordance studies. Von Hofsten and Rönnqvist (1988) presented 5-, 6-, 9-, and 13-month-old infants with three different-sized, moving objects. When infants grasped these objects, the opening and closing of their hand was monitored by measuring the change in distance between thumb and index finger. Nine- and 13-month-olds adjusted the opening of their hand to the target size, in contrast to 5- and 6-month-olds. Clifton, Rochat, Litovsky, and Perris (1991) found similar results with 6.5-month-old infants. They presented infants with two sound-making objects of different sizes, first in the light and then in the dark. Sound cued the objects' identity. In both the light and the dark conditions, the infants adjusted their arm preparation according to the size of the objects. These two studies suggest that infants perceived the width relation between an object and their own system. This width relation specified grasping, which can be seen as a combination of passing through and support. However, the studies do not provide information about infants' perception of width relations between objects that specify passing through and support.

Evidence for infants' perception of the width relation that specifies passing through was provided by a control experiment from a study on infants' sensitivity to solidity and continuity constraints (Spelke, Breinlinger, Macomber, \& Jacobson, 1992). Infants were shown a surface with a gap. This surface was located a few centimeters above the floor of the display. Infants were familiarized with a ball that fell behind a screen and reappeared on the floor of the display, as if it had passed through the gap. Infants were then tested with the same event using two balls of novel sizes: one was small enough to fit through the gap, the other was too large. A lawful test event involved the smaller ball that fell behind the screen and reappeared on the floor of the display. A violation test event involved the large ball that fell behind the screen and reappeared on the floor of the display, as if it had passed through the gap in a miraculous way. Infants from 3.5 months of age looked significantly longer to the violation event than to the lawful event, suggesting that they perceived that the width relation between the ball and the gap did not allow for passing through.

On the basis of findings of these previously described studies, we expected that, between 4 and 9 months of age, infants begin to perceive whether the width relation between a block and a box specifies passing through or support. In the present study, the experiments were designed in the following way: Each trial started with a block approaching a box. In a passing through experiment, infants were familiarized to several instances of a block that passed through the opening of a box. In a support experiment, infants were familiarized to blocks that became supported by the rims of boxes. Block and box width were varied in both the passing through and support experiments to ensure that familiarization occurred to the invariant width relation that specified the event outcome, irrespective of the particular objects involved in the event.

After familiarization, the objects were rearranged into two test pairs. One test pair had a width relation similar to and the other had a width relation different from that seen in the familiarization trials. For the test pair with the similar width relation, the event outcome (a) was similar to that seen in the familiarization trials and (b) conformed lawfully to the outcome specified by the width relation. For the test pair with the different width relation, the event outcome (a) was similar to that seen 
in the familiarization trials but (b) violated the outcome specified by the width relation. Each test pair was shown twice. The four test trials were presented on alternate trials counterbalanced across participants. If infants are able to perceive which outcome is specified by the width relation, a longer looking time would be predicted for an event outcome that violates this specification.

\section{Experiment 1: Passing Through}

\section{Method}

Sample. Fifty-six infants participated in this experiment. Eighteen infants were 4 months old (mean age $=4$ months 4 days), 19 infants were 6 months old (mean age $=6$ months 7 days), and 19 infants were 9 months old ( mean age $=9$ months 3 days). Eight additional infants were excluded from the experiment, 3 because of emotional distress, 2 because of equipment failure, and 3 because their caretakers did not follow the instructions given by the experimenter. Names and addresses of the participants were obtained from the municipal government in Nijmegen, The Netherlands, and parents were compensated for participation.

Apparatus. Events consisted of blocks that were lowered into the opening of boxes and lifted out of them again. These events were displayed behind a window $(57 \times 40 \mathrm{~cm})$ of a display cabin. The window could be occluded by using a black window blind that was operated by an experimenter with the aid of a cord. Changes of displays took place behind the occluded window blind. The blocks were hung on nylon strings, and their motions were controlled by the experimenter with the aid of a handle. The nylon strings were guided by two steel conductors that prevented the block from swaying back and forth. These conductors were not visible to the infants. The blocks moved at a constant speed of $20 \mathrm{~cm} / \mathrm{s}$ and were controlled by a regulator. The timing of the lowering, raising, and holding of the blocks and the changing of the objects were indicated by a computer-controlled monitor.

Objects. Real objects were used, thus allowing visual exploration of surfaces extending in depth. In this way, displayed events were more comparable to environmental events (Slater, Rose, \& Morison, 1984). A large and a small concave object (boxes) and a large and a small solid object (blocks) were used. The blocks $(16.5 \times 10 \times 8 \mathrm{~cm}$ and $6.5 \times 10$ $\times 6.5 \mathrm{~cm}$ ) were made of wood and were painted red. The large box $(31$ $\times 10 \times 20 \mathrm{~cm})$ had an opening of $26 \times 15 \mathrm{~cm}$, and the small box $(20 \times$ $10 \times 20 \mathrm{~cm}$ ) had an opening of $15 \times 15 \mathrm{~cm}$. Both boxes were made of semitransparent plastic. They were dappled randomly with yellow spots to provide obvious texture and to emphasize their substantiality without losing their transparency. The inner back side of each box was covered with black cardboard to emphasize the width of its concavity. Each of the boxes had a rim of $2.5 \mathrm{~cm}$ around the opening. The small block fitted into the opening of the small box as well as into the opening of the large box. The large block apparently fitted only into the opening of the large box. However, the rims on the sides of the small box were partly flexible, making possible the passing of the large block through the opening of the small box. In the test trials, whenever the large block moved downward in the direction of the small box and touched the surfaces of the flexible parts of the side rims, these parts were pushed downward by the block and the block entered the opening of the box. The entering of the block gave the illusion that the block was penetrating the opening through the rim. When the block was raised again, springs underneath the flexible parts of the side rims pushed the parts into their original position.

The dapples on the box concealed the joints of the flexible parts of the rims. The flexible parts of the side rims matched precisely the depth of the large block. Therefore, the outline of the downward and upward moving block concealed the downward and upward motion of these flexible parts. This ensured the illusion of the block penetrating the opening through the rim. Precise matching of the flexible parts of the rim with the depth of the block ensured that the block entered the box in straight motion without jiggling.

Design. Infants were familiarized to a downward motion of a block through the opening of a box and a subsequent upward motion of the block to its original position. This event was considered one cycle; one or more complete cycles occurred in a trial. The number of cycles shown depended on the infants' looking time. Passing through was shown for a downward motion of the small block into and out of the opening of the small box and for a downward motion of the large block into and out of the large box (see left side of Figure 1). Variations in block-box combinations on alternate trials allowed the extraction of the invariant width relation that specifies passing through, irrespective of the particular objects in a trial. After familiarization, the objects were rearranged into two test pairs. One test pair consisted of the small block and the large box. In this test pair again, the width relation specified passing through, similar to the width relation in the familiarization trials. The other test pair consisted of the large block and the small box. In this test pair, the width of the large block apparently did not fit into the opening of the small box. Therefore, support was implied. The relation between the block and the opening of the box was different from the relation shown in the familiarization trials. However, as a result of the flexible parts of the side rims of the box, the motion of the block resulted in passing through.

For the test pair with the similar width relation, the event outcome (a) was similar to that seen in the familiarization phase and ( $b$ ) conformed lawfully to the outcome specified by the width relation between the block and the opening. For the test pair with the different relation, the event outcome (a) was similar to that seen in the familiarization phase but (b) violated the outcome specified by the width relation between block and opening. Each test pair was shown twice. The four test trials were presented on alternate trials, counterbalanced across infants.

Procedure. The procedure was the same for all ages. Infants were tested while sitting on their caretaker's lap in front of the window of the display cabin. The eye level of the infant was adjusted by the height of the caretaker's seat. The infants were seated approximately $70 \mathrm{~cm}$ from the window and looked at an angle of $40^{\circ}$ into the opening of the box. Caretakers were informed about the procedure and were instructed not to look at the displays. If they looked at the displays, their infants were excluded from the experiment.

The durations of the looks of the infants, as indicated by corneal reflection, were scored in tenths of seconds by an observer who observed the infant through a peephole of the display cabin. The observer was not visible to the infants. The durations were recorded with the aid of a button box connected to an Apple II plus computer. The observer was carefully trained but was unaware of the specific object combinations shown on a trial.

Twenty infants were viewed by two observers to determine interobserver reliability. Interobserver reliability, on 0.5 -s intervals of total looking time over trials, averaged $96 \%$.

A variant of an infant-controlled familiarization of the visual looking time task was used (Horowitz, Paden, Bhana, \& Self, 1972). The beginning of each trial was signaled by a tone that was also the sign for the experimenter to lift the black window blind. On each trial, the passing through event involving one of the two block-box combinations was shown for one or more cycles. At the start of each cycle, the distance between the bottom of the block and the edge of the opening of the box was $36 \mathrm{~cm}$. The block reached the bottom of the box in approximately $1.9 \mathrm{~s}$. After $2 \mathrm{~s}$, the block was raised in $1.8 \mathrm{~s}$ to its original position, where it hung for $1.5 \mathrm{~s}$. Thereafter, the same 7.2-s cycle was repeated. A trial ended at the end of the cycle in which the infant looked at the event 

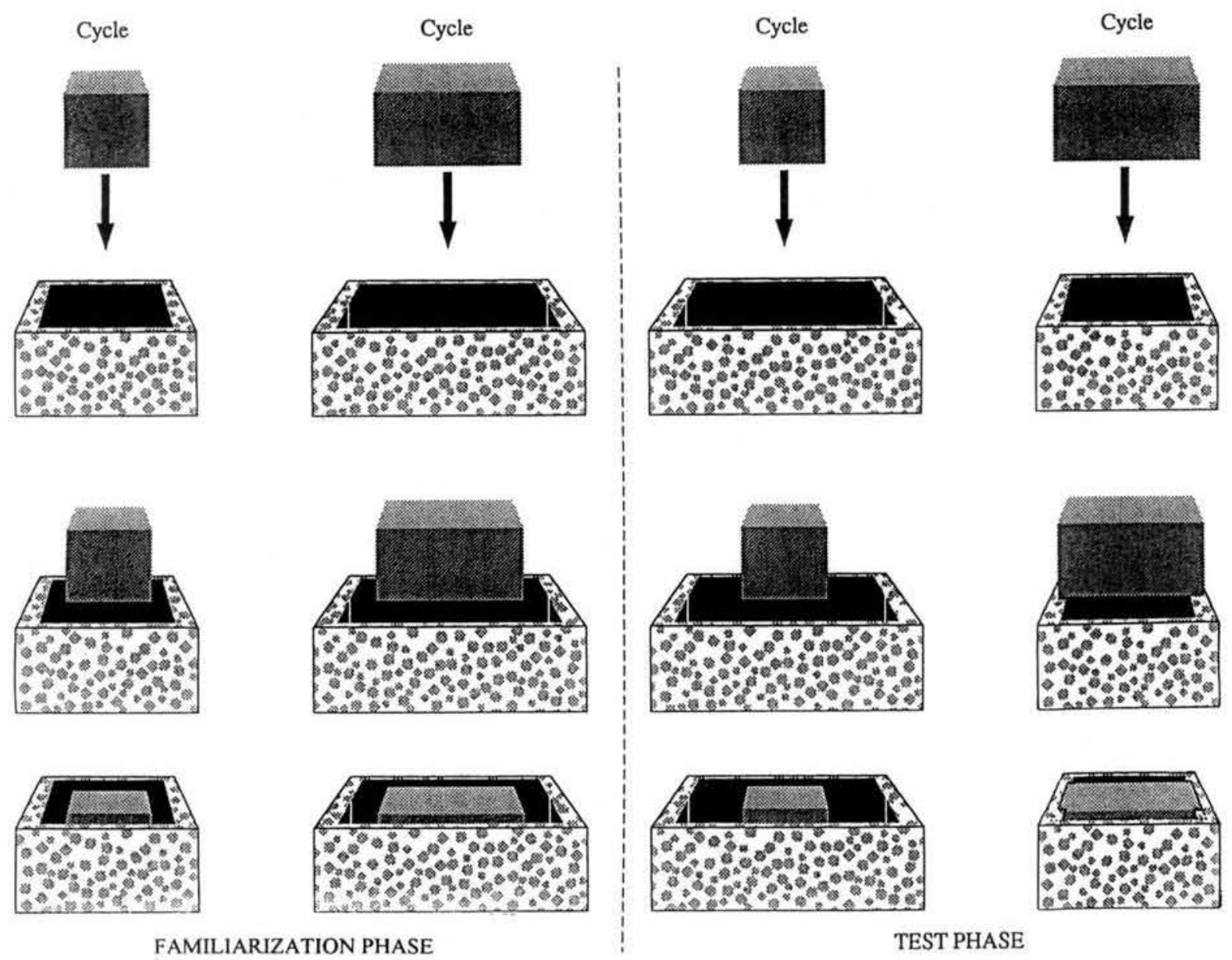

Figure 1. Schematic representation of the familiarization and test displays in the passing through experiment.

for at least $2 \mathrm{~s}$ and had then continuously looked away for $2 \mathrm{~s}$. The end of the trial was signaled by a tone, which was the signal for the experimenter to slide the blind across the window. The blind occluded the display for $4 \mathrm{~s}$. The next trial started with the other combination of objects. Familiarization trials continued until the infant met the criterion of a $50 \%$ or greater decrease in looking time on two consecutive trials in relation to the looking time on the first two trials or after a maximum of 13 familiarization trials. The computer calculated when the infant met the criterion. After familiarization, the test trials were shown using the identical procedure from the familiarization trials with the exception that the block-box combinations were changed (see Figure 1).

\section{Results and Discussion}

Familiarization. The mean number of familiarization trials to reach criterion was 5 for the 9-and 4-month-olds and 6 for the 6-month-olds. Only 2 infants, both 4 months old, reached the maximum of 13 familiarization trials.

Looking times. The infants' looking times during these trials were compared by means of a $3 \times 2 \times 2$ mixed model analysis of variance (ANOVA) with age ( 4,6 , and 9 months old) and order (violation or lawful test trial first) as the betweensubjects variable and test pair (violation or lawful) as the within-subject variable. The analysis revealed significant main effects of age, $F(2,50)=3.98, p<.05$, and of test pair, $F(1$, $50)=30.34, p<.001$. These main effects were qualified by a significant Age $\times$ Test Pair interaction, $F(2,50)=3.67, p<$ .05 . There were no order effects. Further analysis with Tukey's multiple range test revealed the following effects: The 4-monthold infants did not show significantly longer looking times in the violation test trials than in the lawful test trials, whereas the 6and 9-month-olds did show significantly longer looking times in the violation test trials than in the lawful trials (see Figure 2).

These results indicate that in contrast to the 4-month-old infants, the 6-and 9-month-old infants found the passing through outcome in the violation trials much more interesting than the outcome in the nonviolation trials. Because the objects shown in the violation trials were already seen in the familiarization trials, longer looking times could not be ascribed to change in objects. Second, the change of the rim in the violation trials was not visible and thus could not be the reason for longer looking times. Only the width relation between the block and the open- 
ing of the box was changed. Therefore, the data suggest that the infants perceived that this width relation did not specify passing through and looked longer when this outcome resulted. The negative findings of the 4-month-olds are in contrast with the results obtained by Spelke and her colleagues (Spelke et al., 1992). In that study, 3.5-month-old infants perceived that a ball with a width larger than a gap in a surface could not pass through that gap. A possible explanation for this contrasting finding is that the 4-month-olds in Spelke's experiment perceived the violation of width relation because it was more obvious than the violation in our experiments. In our experiment, the difference between the width of the block and the width of the opening was only $1.5 \mathrm{~cm}$, whereas in Spelke's experiment the difference between the width of the ball and the width of the gap was $6 \mathrm{~cm}$. Combination of Spelke's results with ours suggests that infants younger than 4 months of age are able to perceive obvious violations of width relation. Older infants are able to perceive more subtle violations.

The 6- and 9-month-old infants looked significantly longer whenever a block wider than the width of the opening of the box moved into the opening. However, it is not clear whether the infants anticipated that this width relation specified support. Maybe they had no other expectation about the event outcome than that the block would not pass through the opening of the box. To determine whether infants are able to perceive the width relation that specifies support, we performed a second experiment. This experiment investigated infants' ability to perceive the width relation that specifies support. Because of the design of our experiments, passing through and support are complementary event outcomes. The underlying relation is a width relation. When the relation surpasses a critical value, the outcome will be no longer passing through but support. Do infants perceive when the width relation specifies support?

\section{Experiment 2: Support}

\section{Method}

Sample. Participants were 58 infants, similarly recruited as in Experiment 1 . Twenty-one infants were 4 months old (mean age $=4$ months 10 days), 18 infants were 6 months old (mean age $=6$ months 7 days), and 19 infants were 9 months old (mean age $=9$ months 3 days). An additional 7 infants were excluded from the experiment, 1

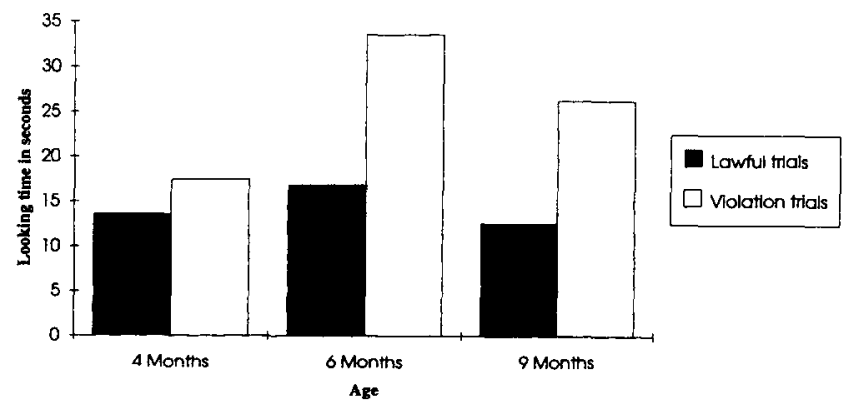

Figure 2. Test pair scores for the three age groups in the passing through experiment. because of his extremely outlying looking scores that caused the distribution of the looking time scores to deviate from normality, 2 because of emotional distress, 2 because of equipment failure, and 2 because their caretakers did not follow the instructions given by the experimenter.

Apparatus. The apparatus was identical to that of Experiment 1 with one exception. After $36 \mathrm{~cm}$, the motion of the block was stopped by a piece of wood, $10 \mathrm{~cm}$ in height, placed under the handle.

Objects. Another large and small box and the same blocks of Experiment 1 were used (see Figure 3). The rims of the boxes were $1 \mathrm{~cm}$ in width. The large box $(15 \times 10 \times 15 \mathrm{~cm})$ had an opening of $14 \times 14 \mathrm{~cm}$. The small box $(8 \times 10 \times 15 \mathrm{~cm})$ had an opening of $7 \times 14 \mathrm{~cm}$. Both boxes were made of the same material as the boxes in Experiment 1.

Design. Infants were familiarized to events that consisted of a block that was lowered and supported by the rims of a box and then raised to its original position. This event was shown for one or more cycles in a trial, depending on the infants' looking times. Support was shown for the small block by the small box and for the large block by the large box. Variations in block-box combinations on alternate trials allowed perception of the invariant width relation that specified support irrespective of the particular objects shown in a trial (see left panel of Figure 3).

After familiarization, the objects were rearranged into two test pairs. One test pair consisted of the large block and the small box. In this test pair, the width relation specified support similar to the width relation in the familiarization trials. The other test pair consisted of the small block and the large box. In this test pair, the outline of the small block did fit into the opening of the large box specifying passing through. The width relation was different from the width relation shown in the familiarization trials. However, because of the piece of wood underneath the handle, the outcome depicted was support. For the test pair with the similar width relation, the event outcome (a) was similar to that seen in the familiarization phase and (b) conformed lawfully to the outcome specified by the width relation between the block and the opening of the box. For the test pair with the different relation, the event outcome (a) was similar to that seen in the familiarization phase but (b) violated the outcome specified by the width relation between the block and the opening of the box. Each test pair was shown twice with the four test trials presented on alternate trials, counterbalanced across infants.

Procedure. The procedure was identical to that used in Experiment 1 , but this time support was shown. At the beginning of each cycle, the distance between the bottom of the block and the opening of the box was $36 \mathrm{~cm}$. The block reached the top level of the box in approximately $1.5 \mathrm{~s}$. After $2 \mathrm{~s}$, the block was lifted in $1.8 \mathrm{~s}$ to its original position, where it hung for $1.5 \mathrm{~s}$. Thereafter, the $6.8-\mathrm{s}$ cycle was repeated. As described earlier, the violation trials consisted of the small block becoming "miraculously" supported by the opening on top of the large box. Placing the piece of wood beneath the handle made the block suddenly stop in midair above the opening on top of the box. The piece of wood was placed beneath the handle throughout the whole experiment to ensure that there was a contact sound when the small block stopped above the opening of the large box in the violation trials. This sound was the same as the sound heard when the block collided on the edge of the box during the lawful test trials and familiarization trials. Therefore, longer looking times could not be ascribed to absence of contact sound in the violation trials or difference in sound among trials. Note that because the sound created the illusion that the block hit a surface, there was contrasting visual and auditory information in the violation trials.

\section{Results and Discussion}

Familiarization. The mean number of familiarization trials to reach criterion was five for all age groups. 


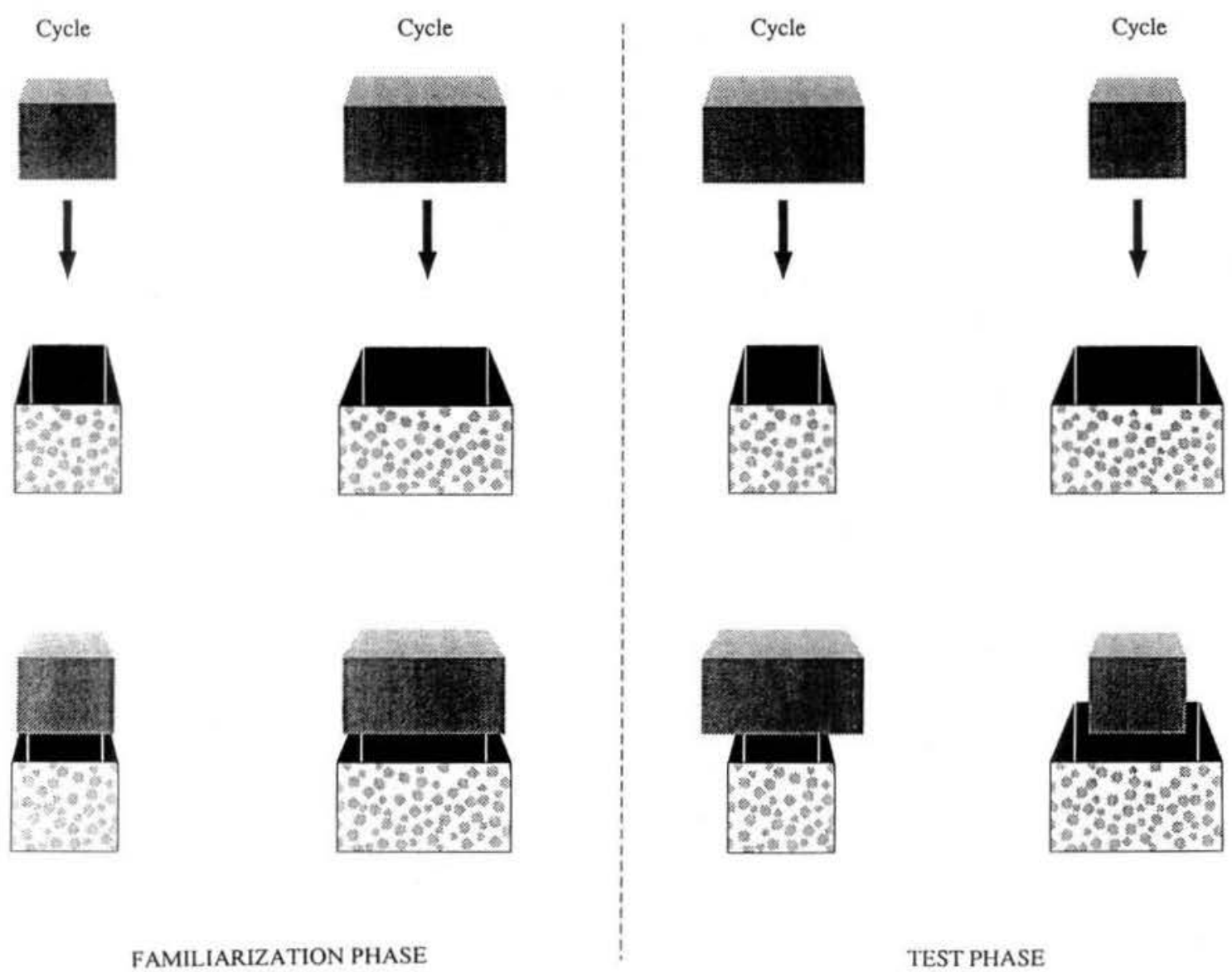

Figure 3. Schematic representation of the familiarization and test displays in the support experiment.

Looking times. The infants' looking times during these trials were compared by means of a $3 \times 2 \times 2$ mixed-model ANOVA with age (4, 6 , and 9 months) and order (violation or lawful trial first) as the between-subjects variables and with test pair (violation or lawful) as the within-subject variable. The analysis revealed a significant main effect of age, $F(2,52)=$ $3.17, p<.05$, and test pair, $F(1,52)=27.18, p<.001$. There was no effect for order. The main effects were qualified by a significant two-way interaction of Age $\times$ Test Pair, $F(2,55)=$ $8.73, p<.001$. Further analysis with Tukey's multiple-range test revealed the following effects: Four-month-old infants did not have significantly longer looking times in the violation test trials than in the lawful test trials. Even though 6-month-olds looked longer at the violation trials than at the lawful test trials (see Figure 4), this difference was not significant. Nine-month-old infants did show such a significant difference.

In the same line of reasoning as in Experiment 1, the results suggest that the 9-month-olds perceived that the width relation did not allow for support and therefore looked longer when support occurred nevertheless. We may conclude that 9-montholds are able to perceive the invariant width relation between a block and box that specifies support. Evidence for this ability in
4- and 6-month-old infants was not found, although the results of the 6-month-olds showed a trend in this direction.

The results of the support experiment for the 6-month-olds contrasted with those for the passing through experiment at this age. The reason for this discrepancy is not clear. One explanation may be found in the specific ways the different events were displayed. Passing through involved a continuation of motion;

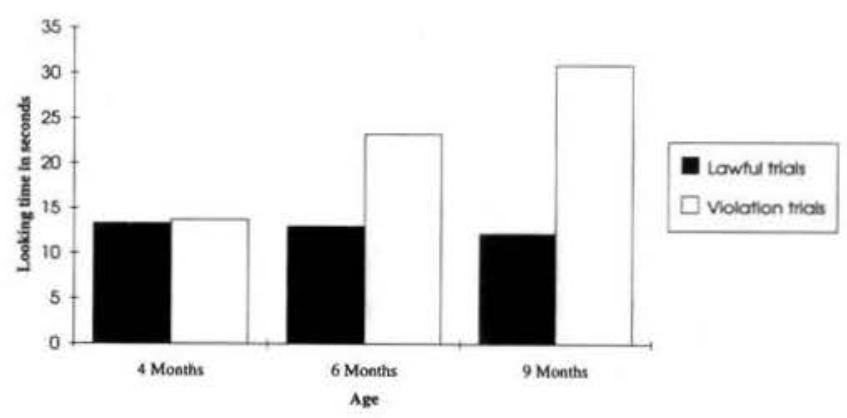

Figure 4. Test pair scores for the three age groups in the support experiment with the contact sound. 
support involved an abruptly halted motion of the block that was accompanied by a contact sound. Although a contact sound implies impact on a surface, Spelke's (Spelke, Smith-Borm, \& $\mathrm{Chu}, 1983$ ) research on 4-month-olds infants' perception of moving and sounding objects indicates that such an implication does not have to exist for infants. Spelke presented infants with two moving objects, side by side, accompanied by a single percussive sound played from a central location. To determine what states or transformations of an object were perceived to be related to sound, Spelke showed infants events in which the sound occurred when one object moved through a particular spatial position, when it abruptly changed its direction, or when it made contact with a rigid surface. Infants perceived the sound and object to be related whenever the sound occurred as the object changed direction, irrespective of its impact with a surface. For 4-month-old infants, contact sound did not necessarily imply support.

Spelke et al.'s (1983) results suggested that in infancy, perception of auditory-visual relations depends in part on detection of discontinuity in the motion of a visible object. This finding raises the possibility that infants might have paid attention to the relation between sound and the halted motion of the block, instead of the width relation. Furthermore, Bushnell and Boudreau (1991) and Bower (1989) suggested that properties that are multimodally specified gain attentional advantage over properties that are specified for only one modality. In the support experiment, the abrupt change in motion was visually and auditorily specified, whereas the width relation was only visually specified. This explanation is strengthened by the findings that infants seem to coordinate an auditory event with a visual event that is appropriate with it and ignore an inappropriate visual event (Spelke, 1976). In Experiment 2, the auditory event was the contact sound, and the visible event that was in accordance with this event was the stopping of the motion of the block. The inappropriate visual event shown was the violation of the width relation. Six-month-old infants may have ignored the violation of width relation and instead may have paid attention to the stopping of the block. Because all of the test trials in the support experiment consisted of a moving block that stopped when a sound was heard, there may have been no difference between the test trials from the infants' point of view.

A second explanation for the discrepant results of the 6month-old infants may be that to 6-month-old infants it might seem that the floating block in the violation trials of the support experiment is resting on the back rim of the box (see Figure 3 ). Although visual depth cues specify that the block is in front and not on top of the rim, the image that the block is resting on the back rim of the box may be strengthened by the contact sound that is heard when the motion of the block stops. Even though the back rim could not provide adequate support for the block, infants 6 months of age may not yet be able to perceive this and, therefore, may not have been surprised when the block stopped at the level of the opening in the violation trials.

In addition to the explanation of perception of width relation, there may be another explanation for the longer looking times of the 9-month-olds in the violating support event. Nine-month-olds may have looked longer at the violation support trials because they perceived auditory information for contact with a surface but did not see such a surface. If this is true, the infants may not have paid attention to the width relation, and thus the explanation of longer looking times caused by perception of violated constraints set by the width relation is no longer valid.

To test whether auditory information drew the attention of 6-month-old infants away from support toward discontinuity in motion or strengthened the image of support by the back rim of the box, we ran a control experiment. This experiment also investigated whether 9-month-olds looked longer at the support trials because of the contradictory visual and auditory information. The control experiment was exactly the same as the support experiment with the exception that contact sound was absent during all trials. We also included 4month-old infants in this experiment. Even though Experiment 1 and 2 showed no evidence for the perception of width relation by the 4 -month-olds, we might not have elicited the infants' best performance. It might be that these infants did not perceive the violation in the support trials for the same reasons as we gave for the absence of this perception in the 6month-olds. On the basis of the data of the 6- and 9-montholds in Experiments 1 and 2, we expected that in this experiment 6-and 9-month-old infants will look significantly longer to the violation than to the lawful test trials. We did not expect such a significant difference for the 4-month-olds.

\section{Experiment 3: Support Without Contact Sound}

\section{Method}

Sample. Participants were ten 4-month-old infants ( mean age $=4$ months 5 days), ten 6 -month-old infants (mean age $=6$ months 2 days), and ten 9-month-old infants (mean age $=9$ months 4 days). Infants were similarly recruited as in Experiment 1.

Apparatus and objects. The apparatus was similar to the one used in the support experiment (Experiment 2 ) with one exception. To delete the sound that originated whenever the handle collided on the piece of wood placed beneath it, we placed a piece of foam on top of the piece of wood $9.5 \mathrm{~cm}$ in height. The objects used in this control experiment were identical to the ones used in the support experiment with one exception. To dismiss the sound that originated whenever the blocks collided with the boxes, the lower part of the blocks was made of foam. The blocks were wrapped up in tape and painted red. This made the foam no longer visible and the blocks looked like solid units.

Design and procedure. The design and procedure were similar to the ones of Experiment 2 (see Figure 3 ).

\section{Results and Discussion}

Familiarization. The mean number of familiarization trials to reach criterion was five for all age groups.

Looking times. The infants' looking times during these trials were compared by means of a $3 \times 2 \times 2$ mixed-model ANOVA with age $(4,6$, and 9 months) and order (lawful or violation trial first) as the between-subjects variable and test pair (violation and lawful) as the within-subject variable. The analysis of the data revealed a significant effect of age, $F(2,23)$ $=6.63, p<.005$, and test pair, $F(1,23)=20.11, p<.001$. Further analysis with Tukey's multiple-range test revealed the following: Six- and 9-month-old infants showed significantly 


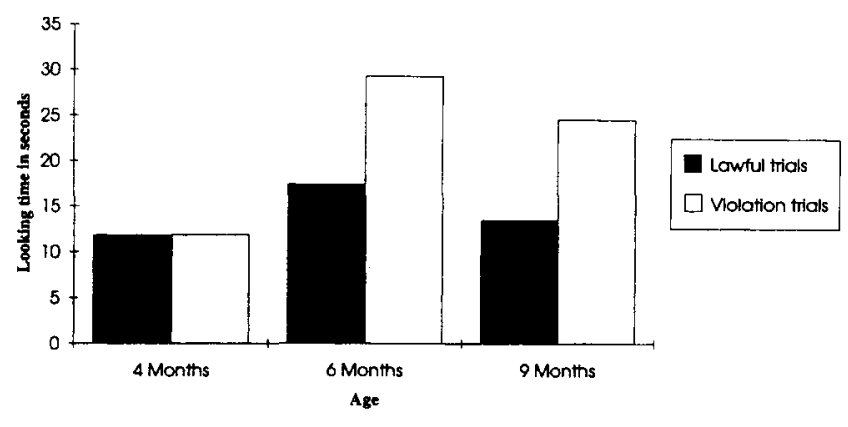

Figure 5. Test pair scores for the three age groups in the support experiment without contact sound.

longer looking times in the violation test trials than in the lawful test trials, whereas 4-month-olds did not show a significant difference in looking times (see Figure 5).

The experiment did show that when contact sound was deleted, infants from 6 months of age were able to anticipate support. On the basis of the data from this experiment, we may also conclude that 9-month-olds looked longer at the violation test trials not because of the contradictory information in these trials but because they perceived the violation of the constraints set by the width relation between the objects. The data did not demonstrate whether sound drew the 6-month-olds' attention away from support toward discontinuity in motion or whether sound strengthened the image of support provided by the back rim of the box. Even though sound was deleted, 4-month-olds still did not look longer at the violation trials. The data suggest that 4-month-old infants did not perceive the width relation between the block and the box. However, another interpretation is possible. To perceive the violation in the support experiment, infants have to (a) perceive that the width relation in the violation trials does not specify support and (b) expect a downwardmoving object to continue its motion until it contacts another surface. Spelke (Spelke et al., 1992) concluded that it is between 4 and 6 months of age that infants start to expect that objects continue to move downward until they reach another surface. Given that 4-month-olds may not understand this principle, absence of this insight, rather than the inability to perceive width relation, may have been the cause of the negative results of the 4-month-olds in the support experiments.

A question that is difficult to answer is whether the older infants anticipated support on the basis of perception of width relation or on the basis of the availability of any supporting surface. However, these two possibilities are intertwined in our experiments, because a width relation that underlies support always provides a supporting surface. Therefore, we cannot answer this question on the basis of our results. It may be that according to 6- and 9-month-old infants, any amount of support by the rims of the box (e.g., by only part of one side rim) is sufficient for support. Further research is needed to answer this question.

In summary, 9-month-old infants looked significantly longer at the violation test trials than at the lawful test trials in the passing through experiment and in the support experiment.
Six-month-olds looked significantly longer at the violation trials in the passing through experiment and in the support experiment in which the contact sound was deleted. These results suggest that between 4 and 6 months of age, infants become able to anticipate event outcomes on the basis of perception of width relation. There is, however, another possible interpretation for the infants' looking preference for the violation test trials. Infants may have looked longer at these trials because the change in width relation caused by the rearrangement of objects provided a relatively larger change in visual layout for the violation test pair but not so large of a change in the case of the lawful test pair ( see Figures 1 and 3 ). It may be that solely the larger change in layout attracted the infants' attention and that they did not perceive that this change specified another event outcome. If this is the case, we may not speak of perception of information provided by object relations.

Another question that arises with respect to perception of the visual layout is whether infants perceived the change in the rims of the box in the violation trials of the passing through experiment. In these trials, it seemed that the block was penetrating the opening of the box through the rims. As a result, space that was first occupied by the rims became subsequently occupied by the block. This change in space occupation causes a change in layout. This change in layout covaries with the violation of width relation and even has to be perceived in order to perceive this violation. However, an important question to answer is whether infants looked longer in the violation trials because (a) they perceived that the block occupied space first occupied by the rims and that this violated the width relation, or (b) they just perceived the changing layout without perceiving that this change specified the violation of the width relation. This latter alternative would imply that the change in layout was noticed but that the information about the interaction that was provided by this change was not.

To test for these alternative interpretations of the results, we ran another control experiment. In this experiment, there was a comparable change in width relation as in the previous experiments, but this change was irrelevant for the event outcome. In other words, it did not provide any information about the course and outcome of the interaction. The same configurations as in the support experiment were shown (see Figure 3), but this time the boxes were closed. The lid on the box provided a solid surface for support irrespective of the width relation between the block and the box. Therefore, the outcome always resulted in support. In the familiarization trials and in one of the test trials, a block became supported by a closed box with a width smaller than the block. In the other test trial, a block became supported by a closed box with a width larger than the width of the block. We expected no difference in looking times between the test trials. However, no conclusion can be drawn from an absence in response. Therefore, we compared the looking times at the test trials in the present experiment with the looking times at the test trials in Experiment 3.

If we find an Experiment (Experiment 3 vs. Experiment 4 ) $\times$ Test Pair (trials with a large change in layout vs. trials with a small change in layout) interaction, in which the infants in Experiment 3 (large change in layout specified other event outcome) but not in Experiment 4 ( small change in layout spec- 
ified the same event outcome) differentiate between the two test pairs, we may conclude that infants picked up the information specified by the width relation. If so, we can reject the hypothesis that longer looking times are related to perceiving a change in layout without perceiving the information that this change specifies.

\section{Experiment 4: Irrelevant Change in Width Relation}

\section{Method}

Sample. Participants were 19 similarly recruited infants as in Experiment 1 . Nine infants were 6 months old (mean age $=6$ months 2 days), and 10 infants were 9 months old (mean age $=9$ months 4 days). One additional infant was excluded from the experiment because his caretaker did not follow the instructions given by the experimenter.

Apparatus, objects, and procedure. These were identical to those in the support experiment (Experiment 2) with the exception that lids were placed on the boxes and that the blocks became supported by these lids.

Design. Infants were familiarized to events that consisted of a block that was lowered, supported by a box, and then raised up to its original position. This event was shown for one or more cycles in a trial. Support events consisted of a downward motion of the small block on the small closed box and of a downward motion of the large block on the large closed box.

After familiarization, the objects were rearranged into two test pairs. One test pair consisted of the large block and the small box. In this test pair, the layout was similar to that shown in the familiarization trials. A block with a width larger than the box became supported. The other test pair consisted of the small block and the large box. In this test pair, the layout was different from that in the familiarization trials. A block with a width smaller than the width of the box became supported. There was a support relation specified in both test pairs because in all trials there was a supporting surface. For both test pairs, the event outcome (a) was similar to that seen in the familiarization phase and (b) conformed lawfully to the outcome specified by the relation between the surfaces of the objects. Each test pair was shown twice. The four test trials were presented on alternate trials, counterbalanced across infants.

\section{Results and Discussion}

Familiarization. The mean number of familiarization trials to reach criterion was five for both age groups.

Looking times. The infants' looking times during these trials were compared by means of a $2 \times 2$ mixed-model ANOVA with experiment (Experiment 3 vs. Experiment 4 ) as the between-subjects variable and with test pair (large change in layout, with or without change in information for the event outcome vs. small change in layout without change in information for the event outcome) as the within-subject variable. The analysis of the data revealed a significant main effect of experiment, $F(1,34)=29.04, p<.0001$, and of test pair, $F(1,34)=18.56$, $p<.0001$. These main effects were qualified by a significant Experiment $\times$ Test Pair interaction, $F(1,34)=19.34, p<$ .0001 . Further analysis revealed that the 6- and 9-month-old infants in Experiment 3 showed significantly longer looking times in the violation test trials than in the lawful test trials, whereas 6- and 9-month-old infants in Experiment 4 did not differentiate between the test trials (see Figure 6). Furthermore, this analysis showed that there was no significant difference in looking times between the two test trials of Experiment 4 and the looking times of the lawful test trials of Experiment 3, suggesting that the events shown in these test trials were of equal difficulty and interest to the infants.

The hypothesis of significantly longer looking times in the violation test trials solely as a result of the perception of a large change in layout without perceiving the information specified by this change can be rejected. We may conclude that in Experiments 1,2 , and 3 , infants showed significantly longer looking times to changes in layout because they perceived the information specified by these changes.

\section{General Discussion}

Our experiments showed that 6-and 9-month-old infants perceived whether the width relation between a block and the opening of a box specified passing through or support. Four-montholds neither seemed to perceive when the width relation specified passing through nor when it specified support. However, previous research (Spelke et al., 1992) suggests that we did not obtain the infants' best performance. It seems that infants this age are able to detect a violation of a passing through width relation if this violation is more obvious.

An alternative explanation for the negative findings of the 4month-olds in the support experiment may be that these findings are due to a lack of understanding of the gravity principle that objects continue to move downward until they reach another surface, rather than to a lack of perception of width relation. It is also possible that the infants perceived the block in the violation trials as resting on the back wall of the box, even after the contact sound was deleted. Although the back rim did not provide the block with sufficient support, the 4-month-old infants may not yet be able to discriminate between sufficient and insufficient support.

In addition to the findings of Caron et al. (1988), we found that from 6 months of age, infants not only perceive that an opening allows passing through for an object but that they also take into account the width relation between the object and this opening. However, this ability depended on how the information was provided. The 6-month-olds perceived that an object wider than the opening of the box cannot pass through this opening. However, they only perceived that a block with a width smaller than the opening should pass through this opening when contact sound was deleted. The explanations for this finding might be partly the same as the explanations for the negative findings in the support experiments for the 4-month-olds. Maybe the 6month-olds were a little more sophisticated than the 4-montholds. At first, the sound may have strengthened the impression that the block was supported by the back rim of the box in the violation trials. In contrast to the 4-month-olds, this impression might have faded away when we deleted the contact sound. The other possibility we suggested was that because of multimodal specification, infants coordinated the contact sound with the appropriate visible event (the halted motion) and ignored the inappropriate visual event (the violation of width relation). When we deleted the contact sound, both the width relation 


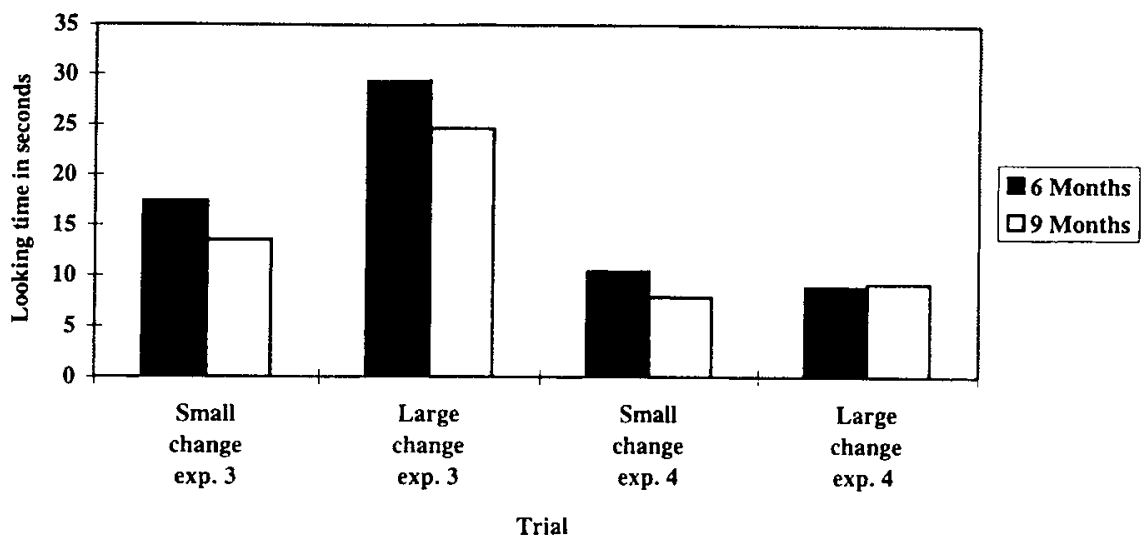

Figure 6. Test pair scores for two age groups in the support experiment (exp.) without contact sound and the experiment with irrelevant change in width relation.

and the contrasting stopping of the motion were only visually specified. Therefore, 6-month-old infants' attention might have been directed toward this contrast, and this may have enabled them to perceive that the width relation did not specify support. The degree to which the first or second explanation accounts for the failure to demonstrate perception of width relation in Experiment 2 could not be abstracted from the results from Experiment 3 and is a matter of further experimentation.

Our data show that between 4 and 6 months of age, infants become able to perceive that a falling object will continue to fall until it contacts a supporting surface. This is consistent with studies on infants' developing knowledge of gravity. Spelke (Spelke et al., 1992) familiarized infants to an event in which a handheld ball was released, falling freely behind a screen and reappearing at rest on the first of two surfaces on its path. In the test trials, that upper surface was removed. The ball fell behind the screen as before, and the screen was raised to reveal the ball at rest either on the lower surface (lawful) or in midair in its familiar position but this time without any support (violation). In contrast to 4-month-old infants, 6-month-old infants looked longer at the violation event than at the lawful event.

In addition to Keil's ( 1979) findings on infants' perception of support, our support experiments showed that around 6 months of age, infants perceive that an object cannot stay in midair without support. Whether infants around this age can distinguish between adequate and inadequate support as Baillargeon and Hanko-Summers (1990) suggested will be investigated in a follow-up study.

On the basis of our results, we cannot say whether infants' ability to perceive width relations must be interpreted in terms of short-term learning or in terms of generalized knowledge about physical relations. An explanation in terms of generalized knowledge seems more plausible. Infants 6 months of age are already confronted with countless experiences of lawful passing through and support events in their daily life. If they are able to learn the relations that underlie our lawful events during familiarization and generalize them to new object combinations, it seems logical that they are also able to learn these relations from their daily experiences and generalize them to new object combinations.

The results show that between 4 and 6 months of age, infants begin to perceive dynamic relations between objects and, therefore, become able to anticipate outcomes of events. This is approximately 6 months earlier than might be expected on the basis of Piagetian theory. According to Piaget's view (Piaget, $1937 / 1954)$, it is not until 12 months of age that infants begin to detect causal relations between objects and not until 18 months of age that they become able to use such relations in means-ends tasks ( see, e.g., Willats, 1989).

Our findings can be interpreted from the Gibsonian (J. J. Gibson, 1979) perspective, which posits that object properties and dynamic relations between them are specified by information that exists as invariances of transformations in the optic array. A width relation that specifies passing through will structure this optic array differently from a width relation that specifies support. For example, when a block wider than the opening of a box approaches this opening from above, at some point in time, it will start and continue to occlude the opening, inside structure and side rims of the box, whereas a block with a width smaller than this opening will not occlude the side rims (see Figures 1 and 3). A follow-up study will investigate whether occlusion patterns provide infants with the information to perceive dynamic width relation between objects.

Although this study was not designed to investigate the information source that enabled infants to perceive width relation, the effect of sound on 6-month-old-infants' ability to perceive support highlights the significance of perceivable information for explaining our results. In addition, this significance is also underscored by the combination of the results of Experiments 3 and 4 ; infants did not just perceive a change in spatial layout but perceived information for the event outcome specified by this change. In other words, infants' looking times are controlled by information about the distal spatial layout specified by cues in the proximal stimulus and not by the proximal cues themselves.

Perception of dynamic relations is not only important for an- 
ticipation, but it also allows for participation in events. Whenever the infant is able to coordinate the perception of relations between object properties to his or her manipulatory activities, tool use becomes possible. For example, when an infant perceives the relation between the width of an object grasped by hand and the concavity of another object and is able to put the grasped object through space into the concave object, it can use the concave object as a container. The fact that infants are able to use spatial perception for the anticipation of event outcomes does not imply that this spatial knowledge is equally accessible to the motor system of the infants (von Hofsten, 1986). The present research indicates that relations between objects are detected by infants at least as early as a comparable relation of an object and his or her grasping system (von Hofsten \& Rönnqvist, 1988).

The finding that the object relations are perceived by infants as early as affordances, to which they are comparable, is of significance to our understanding of infants' perceptual differentiation of events. Perception of affordances of objects and perception of object relations are presumably codeveloping skills that signify infants' growing ability to differentiate events, the objects they involve, their course and outcome, and the possibilities for action.

\section{References}

Baillargeon, R., \& Hanko-Summers, S. (1990). Is the top object adequately supported by the bottom object? Young infants' understanding of support relations. Cognitive Development, 5, 29-53.

Bower, T. G. R. (1989). The perceptual world of the newborn child. In A. Slater \& J. G. Bremner (Eds.), Infant development (pp. 143-182). Hillsdale, NJ: Erlbaum.

Bushnell, E. W., \& Boudreau, P. R. (1991). The development of haptic perception during infancy. In M. A. Heller \& W. Schiff (Eds.), Psychology of touch (pp. 139-161). Hillsdale, NJ: Erlbaum.

Caron, A. J., Caron, R. F., \& Antell, S. E. (1988). Infant understanding of containment: An affordance perceived or a relationship conceived? Developmental Psychology, 24, 620-627.

Clifton, R. K., Rochat, P., Litovsky, R. Y., \& Perris, E. E. (1991). Object representation guides infants' reaching in the dark. Journal of Experimental Psychology: Human Perception and Performance, 17, 323329.

Day, R. H., \& McKenzie, B. E. (1973). Perceptual shape constancy in early infancy. Perception, 2, 315-320.

Day, R. H., \& McKenzie, B. E. (1981). Infant perception of the invariant size of approaching and receding objects. Developmental Psychology, 17, 670-677.

Gibson, E. J., Owsley, C. J., \& Johnston, J. ( 1978). Perception of invariants by five-month-old infants: Differentiation of two types of motion. Developmental Psychology, 14, 407-415.

Gibson, E. J., Owsley, C. J., Walker, A., \& Megaw-Nyce, J. ( 1979). Development of the perception of invariants: Substance and shape. Perception, 8, 609-619.
Gibson, J. J. (1979). The ecological approach to visual perception. Boston: Houghton-Mifflin.

Horowitz, H. D., Paden, L., Bhana, K., \& Self, P. (1972). An infantcontrol procedure for studying infant visual fixations. Developmental Psychology, 7, 90.

Keil, F. (1979). The development of the young child's ability to anticipate the outcomes of simple causal events. Child Development, 50 , 455-462.

Kellman, P. J. ( 1984). Perception of three-dimensional form by human infants. Perception and Psychophysics, 36, 353-358.

Maclean, D. J., \& Schuler, M. (1989). Conceptual development in infancy: The understanding of containment. Child Development, 60 , $1126-1137$

Piaget, J. (1954). The construction of reality in the child (M. Cook, Trans.). London: Routledge \& Kegan Paul. (Original work published 1937)

Pieraut-Le Bonniec, G. (1984, April). Motor-visual integration and the development of the concept of containment. In E. Spelke (Chair), Perception and action. Symposium conducted at the Fourth International Conference on Infant Studies, New York.

Pieraut-Le Bonniec, G. (1985). From visual-motor anticipation to conceptualization: Reaction to solid and hollow objects and knowledge of the function of containment. Infant Behavior and Development, 8, 413-424.

Ruff, H. A. (1982). Effect of object movement on infants' detection of object structure. Developmental Psychology, 18, 462-472.

Ruff, H. A. (1985). Detection of information specifying the motion of objects by 3-and 5-month-old infants. Developmental Psychology, 21, 295-305.

Slater, A., Rose, D., \& Morison, V. ( 1984). Newborn infants' perception of similarities and differences between 2-dimensional and 3-dimensional stimuli. British Journal of Developmental Psychology, 2, 287294.

Spelke, E. S. (1976). Infants' intermodal perception of events. Cognitive Psychology, 8, 553-560.

Spelke, E. S., Breinlinger, K., Macomber, J., \& Jacobson, K. (1992). Origins of knowledge. Psychological Review, 99, 605-632.

Spelke, E. S., Smith-Borm, W., \& Chu, F. (1983). Perception of moving, sounding objects by four-month-old infants. Perception, 12, 719-732.

Stambak, M., Sinclair, H., Verba, M., Moreno, L., \& Rayna, S. (1989). Infants and logic. In H. Sinclair, M. Stambak, I. Levine, S. Rayna, \& M. Verba (Eds.), Infants and objects: The creativity of cognitive development (pp. 15-59). San Diego, CA: Academic Press.

Uzgiris, I. C. ( 1972). Patterns of cognitive development in infancy. Merrill-Palmer Quarterly of Behavior and Development, 19, 181-203.

von Hofsten, C. (1986). Early spatial perception taken in reference to manual action. Acta Psychologica, 63, 323-335.

von Hofsten, C., \& Rönnquist, L. (1988). Preparations for grasping an object: A development study. Journal of Experimental Psychology: Human Perception and Performance, 14, 610-621.

Willats, P. (1989). Development of problem solving in infancy. In A. Slater \& J. G. Bremner (Eds.), Infant development (pp. 143-182). Hillsdale, $\mathrm{NJ}$ : Erlbaum.

Received June 20, 1992

Revision received April 21, 1994

Accepted April 21, 1994 